\title{
Height measurements to diagnose short stature and obesity during after-hours period are inefficient.
}

\section{Takako Matsumoto}

Kurume University School of Medicine

Kikumi Ushijima-Fuchino

Kurume University School of Medicine

Tamotsu Fujimoto

Kurume University School of Medicine

Shuichi Yatsuga ( $\nabla$ bluemif@gmail.com )

Kurume University School of Medicine

\section{Research Article}

Keywords: short stature, obesity, after-hours pediatric examination

Posted Date: January 18th, 2022

DOI: https://doi.org/10.21203/rs.3.rs-1251715/v1

License: (c) (i) This work is licensed under a Creative Commons Attribution 4.0 International License. Read Full License 


\section{Abstract}

Background: Pediatricians in Japan usually measure child body weight during medical examinations. However, they rarely measure child height. Furthermore, during examinations at night or on holidays they may not measure child height at all. There are no reports addressing the efficiency of pediatric height measurement during medical examinations, especially those taking place at night or on holidays. Our study asked whether height measurements and obesity index calculations during all pediatric visits were efficient.

Methods: We analyzed data collected on patients between the ages of 3 and 10 years who visited a pediatrics department over a 1-month period. We divided the patients into four groups based on when they visited the department: 1) weekday-day, 2) weekday-early night, 3) weekday-late night, 4) holidayday. The height and the body weight of all patients were measured. This analysis included 1101 patients. The numbers of patients visiting the hospital were $727,176,34$, and 164 for daytime, early night, late night, and holiday visits, respectively.

Resalts: There were no significant differences in ages of children visiting at different times $(p=0.57)$. However, there were significant differences in height variation among children visiting at different times $(p=0.008)$, with the late-night group being the most variable. There were no significant differences in the incidence of obesity (depend on body mass index (BMI)) among patients visiting at different times $(p=0.44)$; however, significant variations were found for BMI among the patients, $(p=0.03)$, being significantly lower for the late night group than the others.

Conclusions: We conclude that using height measurement to detect short stature (SS) and calculating $\mathrm{BMI}$ to detect obesity is inefficient for children who visit hospital during emergency times.

\section{Introduction}

Short stature (SS) is defined as height of -2.0 standard deviations (SD) or less than children of the same sex and age. Height assessment is important, as $5 \%$ of SS cases are treatable, such as in growth hormone deficiency (GHD), Turner's syndrome in females [1], malnutrition, and abuse during childhood. SS is typically found through information from parents, multiple checkups, and visits to pediatrics departments. In Japan, it is common practice for pediatricians to measure child body weight. However, they do not always measure child height during examinations conducted in daytime. Furthermore, pediatricians may not measure child height at all during examinations at night or on holidays. Therefore, cases of SS may be overlooked despite, examination. There are no reports concerning the efficiency of pediatric height measurements at medical examination, especially during nighttime visits or holidays. Our study asked whether pediatric height measurements and obesity index calculations are efficient in the context of patients visiting at different times of the day, and weekdays vs. holidays.

\section{Methods}


We recruited patients analyzed data collected on patients who can keep still for measuring height between the ages of 3 and 10 years who visited the pediatrics department at Oita Children's Hospital between 1st and 28th February 2016. At hospital reception, hospital staff asked guardians about perinatal information (gestational age, birth weight, height of birth, birth order). The height and body weight of all patients were measured by trained nurses. We excluded some patients who could not record their height and/or weight due to sleeping or poor condition to keep standstill or suspicion of air infection.

The Oita Children's Hospital has 40 beds for mainly primary care and secondary emergencies; it does not have an intensive care unit. We divided the patients into four groups based on when they visited the department: 1) weekday-day, 2) weekday-early night, 3) weekday-late night, 4) holiday-day. Weekdays were Monday to Friday; holidays included Saturday, Sunday, and national holidays. Daytime was defined as $9 \mathrm{am}$ to $5 \mathrm{pm}$, early night as $5 \mathrm{pm}$ to $12 \mathrm{pm}$, and late night as $12 \mathrm{am}$ to $9 \mathrm{am}$. Weekday-early night, weekday-late night, and Holiday visits were considered as "emergency" visits. We defined obesity as a body mass index (BMI) above the 95th percentile[2]. Small for gestational age (SGA) is defined as a condition in which birth weight and height are lower than the 10th percentile compared to the anthropometric standard at birth for each trimester of pregnancy.

\section{Statistical analysis}

The normality of the data distribution was analyzed using the Shapiro-Wilk test with alpha set at 0.05 . The parametric continuous variables age and height were presented as means with standard deviations (SDs). Nonparametric continuous variables comprising body weight (in $\mathrm{kg}$ ) and BMI percentile were presented as medians with interquartile ranges (IQR). Between-group comparisons were conducted using the Kruskal-Wallis test and the Steel test (with daytime as the control). Fisher's exact tests analyzed gender, and incidences of short stature and obesity. The data were analyzed using JMP version 13.2.1 for Windows (SAS Institute Inc., USA). All data generated or analysed during this study are included in this published article and its supplementary information files.

\section{Results}

A total of 6205 patients visited Oita Children's Hospital during the study period, including 3449 who were between 3 and 10 years old (55.5\%). The guardians of 2936 patients (85\%) consented to the child's data being used. Data from 1835 patients were excluded due to being incomplete: 947 records were missing the height information, 640 concerned the third visit or more by the same child, 181 contained neither height nor weight information, and 29 were missing an identification number. Data from an additional 11 patients were excluded because the patient was either under 3 years or over 11 years of age, 9 because height was more than 10 SD above the mean, and one because the visit time was not recorded (Fig. 1).

We analyzed data from 1101 patients, of which 555 were male (50.4\%). The numbers of patients visiting the hospital were $727,176,34$, and 164 during daytime, early night, late night, and holidays, respectively. The mean age of patients was $6.18 \pm 2.14$ years. There was no significant age variation among groups visiting the hospital at different times $(\mathrm{p}=0.57)$. 
The data for height SD were normally distributed ( $p=0.016)$ (Fig. 2), with an overall mean of $0.17 \pm 1.13$ SD. The mean height SDs of children visiting at different times were $0.17 \pm 1.29,0.11 \pm 1.08,0.76 \pm 0.73$, and $0.23 \pm 1.18$ for daytime, early night, late night, and holiday, respectively. There were significant differences related to times of visiting $(p=0.008)$. More specifically, the mean value for the late-night group was significantly higher than for the other groups.

The number of patients recorded as obese based on body weight was 55 (4.99\% of 1101$)$. Within this patient group, the numbers visiting during daytime, early night, late night and holidays were $33(4.53 \%$ of Daytime visitors), 13 (7.38\%), 2 (5.88\%) and 7 (4.26\%), respectively. There were no significant differences in the incidence of obesity among patients presenting at different times $(p=0.44)$.

The BMI percentile data were not normally distributed. The overall median BMI percentile was 59.2 (IQR: 36.6-78.8), and 59.9 (IQR: 38.1-79.1) for daytime visits, 64.8 (IQR:37.7-82.95) for early-night visits, 46.3 (IQR:29.9-76.6) for late-night visits, and 53.8 (IQR:32.0-73.5) for holiday visits. There were significant differences among these values $(p=0.03)$. When we compared the groups, the mean value for the latenight group was significantly low (Table1).

Table1. The characteristics of participants for each visiting time.

\begin{tabular}{|c|c|c|c|c|c|c|}
\hline & & \multicolumn{5}{|c|}{ Time of visit } \\
\hline & & Daytime & Early night & Late night & Holiday & $\mathrm{p}$ \\
\hline All & & 727 & 176 & 34 & 164 & 0.97 \\
\hline $\mathrm{F}$ & & 359 & 90 & 17 & 80 & \\
\hline M & & 368 & 86 & 17 & 84 & \\
\hline \multirow[t]{2}{*}{ Age (y) } & mean & 6.28 & 5.95 & 6.25 & 6.01 & 0.14 \\
\hline & SD & 4.54 & 4.78 & 5.15 & 4.47 & \\
\hline \multirow[t]{2}{*}{$\mathrm{Ht}-\mathrm{SDS}$} & mean & 0.16 & 0.11 & 0.76 & 0.23 & 0.008 \\
\hline & SD & 1.38 & 1.08 & 0.73 & 1.18 & \\
\hline \multirow[t]{2}{*}{ BW (kg) } & median & 19.5 & 18.85 & 20.35 & 18.65 & 0.18 \\
\hline & quartile range & 8.2 & 8.18 & 7.95 & 6.87 & \\
\hline \multirow[t]{2}{*}{ BMI percentile } & median & 59.9 & 64.8 & 46.3 & 53.85 & \\
\hline & quartile range & 41 & 45.25 & 46.7 & 41.53 & \\
\hline Short stature & & 22 & 3 & 0 & 4 & 0.774 \\
\hline
\end{tabular}




\section{Discussion}

The results of this study suggested that pediatric height measurements or obesity index calculations at patient visits to evaluate SS and/or obesity during after-hours period were inefficient.

In Japan, due to the universal health insurance system the examination fee is modest and almost the same for visits during emergency and regular times. Non-emergency patients sometimes visit the hospital at various times during the day due to parental anxiety $[3,4]$. Social and economic factors in the family, education, and health care awareness may all increase the incidence of emergency visits[5]. Furthermore, children classified as being in poverty had more emergency time hospital visits [6]. We considered the possibility that a higher proportion of the patients visiting during emergency time had SS due to poor nutrition. We think it might be possible to find children with SS that had previously gone undetected. Our results do not show more children with SS as a function of the time of emergency. We reasoned that the height distributions would be normal for all visiting times. However, the absence of patients with SS during late-night visits might reflect the small number of such visits. With more late-night visits the number of SS cases may also increase. During emergency times hospitals are staffed by a limited number of doctors, nurses, and other personnel. Measurering the height of unwell children when medical resources are limited is inefficient.

We expected that the numbers of obese patients at emergency times would be high, but this was not the case; the BMI distribution appeared normal. Although caution is required in view of the small number of emergency time visits, we also suggest that calculating BMI, given the limited resources available during emergency times is inefficient.

We used perinatal information to detect SGA but found no treatable SGA patients in this study. The prevalence of SGA in Japan is 3.5\% [7], and the estimated percentage of SGA cases that meet the criteria for $\mathrm{GH}$ treatment is $0.06 \%$. We had no treatable SGA cases because of the relatively meager perinatal information available. We would also argue that obtaining perinatal information with a view to detecting SGA during emergency time examinations is inefficient.

There were several limitations to this study. First, we focused on pediatric examinations in a single hospital, for only one month. Because patient characteristics change across the seasons, it would be valuable to extend such a study throughout the year. Similarly, some patient characteristics might vary across geographical regions, and so regional factors should be considered. Second, our study excluded patients under 3 years old and over 10 years old. We intended to measure height exactly with a standing position. So, we do not know if late-night measurements are suitable or effective for patients in those two age categories. Third, only $31.9 \%$ of the target-age patients who visited the hospital had their height measured. We excluded the patients who could not measuring height and/or weight exactly with a standing position. The excluded patients are poor condition, emergency condition, sleeping or suspicion of air infection. Therefore, there might be more cases of SS and obesity patients than we were able to detect. Finally, outcomes may be different depending on how many patients the hospital receives during 
emergency times; a hospital receiving hundreds might produce a different picture than one receiving relatively few.

\section{Conclusions}

The height measurement to detect SS and calculating BMI to detect obesity for children who visit the hospital during after-hours is inefficient.

\section{Abbreviations}

GHD: Growth hormone deficiency, BMI: Body mass index, SD: standard deviations, SGA: Small for gestational age, SS: short stature

\section{Declarations}

The study was approved by the Ethical Committee of the Oita Children's Hospital for pediatrics (approval number 2016-1). All the experiment protocols for involving humans were in accordance with the relevant guidelines and Declaration of Helsinki in the manuscript. Guardians of all patients provided written informed consent.

\section{Consent for publication}

Not appliable.

\section{Competing interests}

The authors declare that they have no competing interests.

\section{Authors' contributions}

SY conceived the study and was involved in the interpretation of the data and refine the manuscript. TF participated in its design and coordination. KU participated in its design and in the study design and collected the data. TM performed the statistical analyses, interpretation of the data, and drafted the manuscript. All authors read and approved the final manuscript.

\section{Funding}

This research did not receive any specific grant from funding agencies in the public, commercial, or notfor-profit sectors.

\section{Acknowledgements}

The authors would like to express their great appreciation to all the children who participated in this study. This work is supported by the DMC Corporation. 
Availability of data and materials

The datasets used and analyzed during the current study are available from the corresponding author on reasonable request.

\section{References}

1. Grote FK, Oostdijk W, De Muinck Keizer-Schrama SM, van Dommelen P, van Buuren S, Dekker FW, et al. The diagnostic work up of growth failure in secondary health care; An evaluation of consensus guidelines. BMC Pediatr. 2008;8:21.

2. Ogden CL, Flegal KM. Changes in Terminology for Childhood Overweight and Obesity. 2010;:6.

3. Murata M, Ueda I, Yamamori A, Adachi S, Ihimaru Y, Ihimaru Y. A Study of the Conditions and Needs of Patients in Pediatric Emergencies: A Questionnaire Survey of Consultations at a Hospital. Kyoto Igaku Zasshi. 2002;49:33-6.

4. TAN Y. Analysis of parents' thoughts regarding their judgments and responses when their children were suddenly taken ill: parents' fear, peace of mind, coping behavior and demands of government in the free-answer question. Nihon Koshu Eisei ZasshiJAPANESE J PUBLIC Health. 2007;54:711-22.

5. Vivier PM, Rogers ML, Gjelsvik A, Linakis JG, Schlichting LE, Mello MJ. Frequent Emergency Department Use by Children. Pediatr Emerg Care. 2019;Publish Ahead of Print.

6. Sato $Y$, Yamaguchi E, Wada H, Takeuchi $H$. Health Status and Family Characteristics of Elementary and Junior High School Students Living in Poor Households: A Multicenter Survey in Outpatient Clinics. The journal of the Japan Pediatric Society.

7. Fujita K, Nagasaka M, Iwatani S, Koda T, Kurokawa D, Yamana K, et al. Prevalence of small for gestational age (SGA) and short stature in children born SGA who qualify for growth hormone treatment at 3 years of age: Population-based study: Prevalence of short stature in SGA. Pediatr Int. 2016;58:372-6.

\section{Figures}




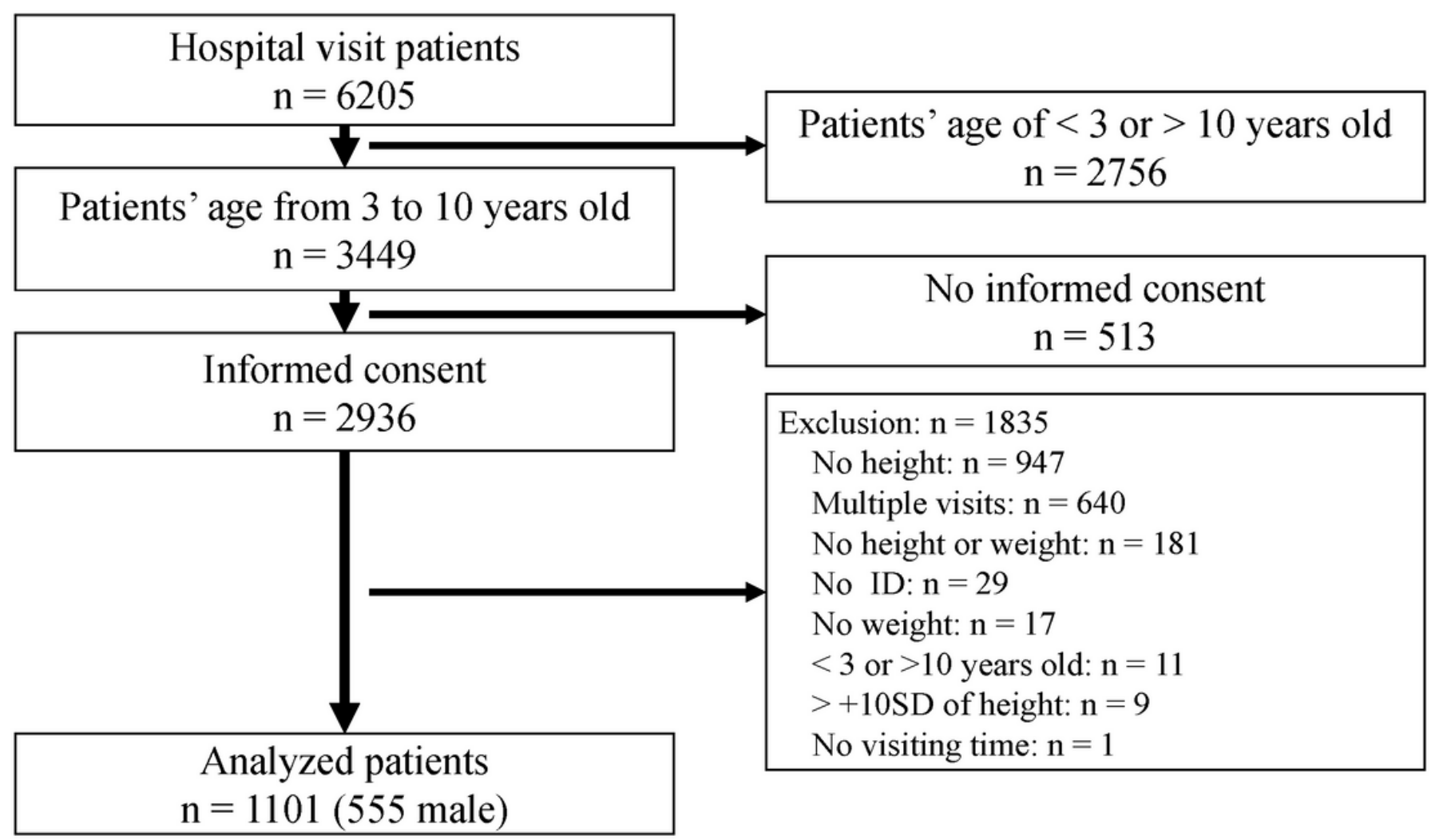

Figure 1

A flow chart of the study participants. ID; identification, n; number, SD; standard deviation.

The distribution of height
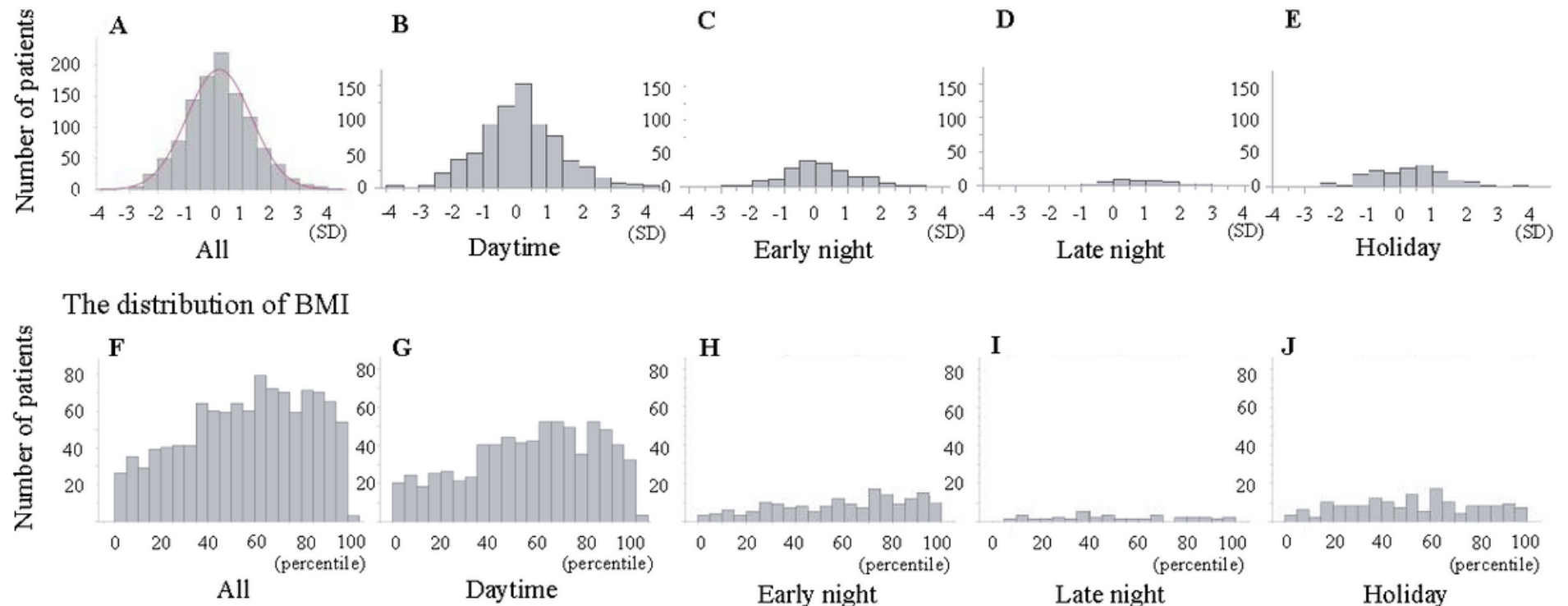

$\mathbf{H}$

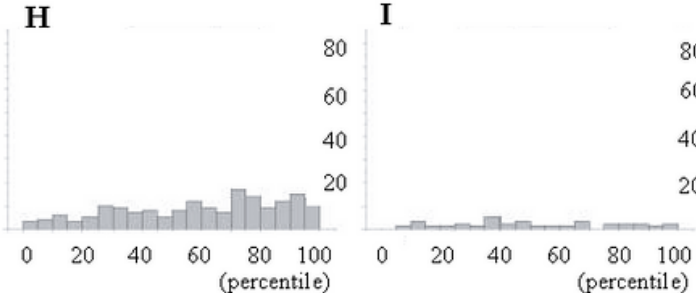

Early night
Late night

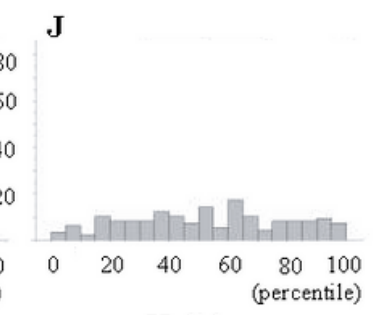

Holiday

Figure 2 
The distribution of height and weight for participants. A-E; the distribution of height-SD, F-J; the distribution of BMI-percentile. BMl; body mass index, SD; standard deviation. 